drug related (ADEs). The effect of clinical pharmacy on ADEs and drug costs has not been substantiated in randomised controlled trials.

Purpose To study the clinical effect of pharmaceutical optimisation of the medication process at admission to hospital.

Materials and Methods Medical patients admitted to three Danish acute wards aged $\geq 18$ years and taking $\geq 4$ types of medicine per day were eligible for inclusion. The patients were randomised to either intervention or control group. A retrospective control group was also formed.

The intervention was comprised of: medicines history, medicines review, medicines reconciliation and entry of proposed prescriptions in the electronic medication system.

Primary endpoint was the proportion of patients with adverse drug events (ADEs), identified by screening the charts for 25 defined triggers from the Danish version of 'Global Trigger Tool'. ADEs were then validated and evaluated for severity scores by two independent panels of clinicians blinded for the intervention.

Secondary end points were length of hospital stay (LOS), inhospital drug costs, readmissions and death within one year of discharge.

Results From March 2010 to July 2011 a total of 1775 patients were screened for inclusion. 573 patients were included, 74 were excluded due to discharge within 24 hours, leaving 499 patients in the study. Preliminary studies clearly showed that the intervention group had fewer ADEs and triggers, shorter LOS and lower inhospital drug costs than the control groups, although no significant differences were recorded.

Conclusions Although the findings did not reach statistical significance, the clinical pharmacist's intervention tended to have a positive impact on the medication process in terms of higher patient safety and lower health care costs.

No conflict of interest.

\section{CPC-112 PREDICTORS OF POTENTIALLY INAPPROPRIATE PRESCRIBING IN ELDERLY FALLERS}

doi:10.1136/ejhpharm-2013-000276.569

D 0'Sullivan, J Carroll, C Meegan. Mater Misericordiae University Hospital, Pharmacy, Dublin 7, Ireland (Rep.)

Background A study to explore the rate of potentially inappropriate prescribing (PIP) in elderly fallers, and the impact of pharmacist-led medicines reviews was undertaken. Data relating to possible predictors of PIP, identified in the literature, were also collected.

Purpose To investigate the following factors as predictors of PIP in elderly fallers:

- Demographic data

- Drugs classes

- Polypharmacy

Materials and Methods The following data were collected as part of a larger study:

- Demographic data: age, gender and days since admission at time of fall

- Number of regular medicines

- Name and class of PIMs identified

\section{Results}

- Sixty patients were included in this study, $34(56.7 \%)$ of whom were male.

- The median age was 79 years (range: 29). Patients were taking a median of 9 regular drugs (range: 17). Twenty-one $(35 \%)$ patients were prescribed $\geq 1$ PIM at the time of their fall.
- Gender was not a predictor of PIP, with 13 male and 8 female patients prescribed $\geq 1$ PIM $(\mathrm{P}=0.548)$.

- Excessive polypharmacy ( $\geq 10$ medications) was identified as a positive predictor of PIP. Participants prescribed $\geq 1$ PIM were taking a mean of 10.86 regular medicines; those not prescribed $\geq 1$ PIM were taking a mean of 7.67 regular medicines $(p<0.001)$.

- A drug from each class in section H of the STOPP criteria was identified at least once. Benzodiazepines were the most frequently prescribed PIM drug class, accounting for $59 \%$ of PIMs overall. Six patients in the baseline group and 7 in the intervention study were prescribed a benzodiazepine. The most commonly prescribed PIM was temazepam.

Conclusions Polypharmacy is a predictor of PIP. Patients prescribed $\geq 1$ PIM were taking on average 3 more regular medicines than those who were not prescribed $\geq 1$ PIM ( $p<0.001)$. Gender was not a predictor of PIP.

No conflict of interest.

\section{CPC-113 PREOPERATIVE ORAL IRON PRESCRIPTION IN THE PREVENTION OF POSTOPERATIVE ACUTE ANAEMIA IN ORTHOPAEDIC SURGERY}

doi:10.1136/ejhpharm-2013-000276.570

${ }^{1}$ A Razurel, ${ }^{1} \mathrm{JV}$ Chauny, ${ }^{1} \mathrm{~B}$ Politis, ${ }^{2} \mathrm{X}$ Dupont, ${ }^{3} \mathrm{D}$ Boyeldieu, ${ }^{4} \mathrm{~F}$ Benhamou, ${ }^{1} \mathrm{~F}$ Le Mercier, ${ }^{4}$ P Hardy. 'Hospital Ambroise Paré, Pharmacy, Boulogne Billancourt, France; ${ }^{2}$ Hospital Ambroise Paré, Anaesthesia, Boulogne Billancourt, France; ${ }^{3}$ Hospital Ambroise Paré, Blood products, Boulogne Billancourt, France; ${ }^{4}$ Hospital Ambroise Paré, Orthopaedic surgery, Boulogne Billancourt, France

Background A previous study on postoperative acute anaemia in orthopaedic surgery was conducted in 2011 (S1), showing that anaemia is recurrent and not always treated. We have recommended prescribing oral iron in the month preceding surgery. A pre-dispensed oral iron prescription has been set up for use during preoperative anaesthesia consultations. We decided to evaluate the impact of this recommendation by a second study in 2012 (S2).

Purpose To assess the impact of preoperative oral iron prescription on the prevalence and treatment of postoperative acute anaemia in orthopaedic surgery.

Materials and Methods Both studies included patients who underwent total hip or knee arthroplasty (THA/TKA). S1 was conducted retrospectively on all patients operated on in 2011. S2 was conducted prospectively on patients who had been prescribed preoperative iron in September/October 2012. We collected data about operations, iron prescriptions, haemoglobin levels, transfusions and lengths of stay.

Results Operations: 327 (S1): 205THA/122TKA vs. 30 (S2): 13THA/17TKA. Postoperative iron prescription: $69 \%$ of patients (S1): oral iron $32 \%$, intravenous iron $20 \%$, both oral and intravenous iron $17 \%$ vs. $43 \%$ of patients (S2): oral iron $23 \%$, intravenous iron $13 \%$, both oral and intravenous iron $7 \%$. Haemoglobin levels: between preoperative and immediate postoperative periods, mean decrease was from $12.9 \pm 0.2 \mathrm{~g} / \mathrm{dl}$ to $11.1 \pm 0.1 \mathrm{~g} / \mathrm{dl}$ (S1) vs. $13.3 \pm 0.2 \mathrm{~g} / \mathrm{dl}$ to $11.7 \pm 0.2 \mathrm{~g} / \mathrm{dl}(\mathrm{S} 2)$, between preoperative period and hospital discharge, mean loss was $2.2 \pm 0.2 \mathrm{~g} / \mathrm{dl}$ (S1) vs. $1.9 \pm 0.17 \mathrm{~g} / \mathrm{dl}(\mathrm{S} 2)(\mathrm{p}<10-3)$. Transfusions: $29 \%$ of patients (S1) vs. $20 \%$ (S2) ( $p<10-3)$. Length of stay: mean was $10.6 \pm 0.8$ days (S1) vs. $8.3 \pm 0.3$ days (S2) $(p<0.005)$.

Conclusions The prospective study showed that oral iron preventive treatment significantly decreases haemoglobin level fall, transfusion rate and length of stay. Therefore it is necessary to sensitise prescribers concerning preventive iron coverage. A further study is needed to evaluate the impact of a longer iron preventive treatment on a larger number of patients.

No conflict of interest. 